(OR 2.55; 95\% CI 0.84 - 7.72); welders (OR 2.98; 95\% CI $0.78-11.40$ ); and drivers of heavy equipment (OR 1.95; 95\% CI 0.66 - 5.81). Reduced risks were found for management and administration jobs (OR 0.70; 95\% CI 0.49 - 0.99); other health care jobs (OR 0.44; 95\% CI 0.20 - 0.99); repairers (OR 0.49 ; 95\% CI 0.22 - 1.09); and electricians (OR 0.46; 95\% CI 0.17 - 1.26).

Conclusions Increased risks for social science jobs could be related to numbers of people contacted in a day and risk of infections. Severe influenza was associated with increased risk of PD but number of people contacted was not. Elevated risks for farmers are typically attributed to pesticide exposure, however our pesticide analyses did not support this attribution. Other exposures of interest for farmers could include animal contact, influenza, head injuries, and vibration from heavy equipment, all associated with PD in our study.

\section{OCCUPATIONAL EXPOSURE TO PESTICIDES AND PARKINSON DISEASE}

${ }^{1} \mathrm{M}$ van der Mark, ${ }^{1}$ Vermeulen, ${ }^{1}$ Huss, ${ }^{2}$ Nijssen, ${ }^{1}$ Kromhout. ${ }^{1}$ RAS, Utrecht, Nederland; ${ }^{2}$ St. Elisabeth Hospital, Tilburg, Nederland

10.1136/oemed-2013-101717.282

Objectives The objective of this study was to assess the risk of occupational exposure to pesticides on the development of Parkinson Disease.

Methods We conducted a multi-centre hospital-based case-control study in the Netherlands. All patients, diagnosed with Parkinson Disease in one of the five participating hospitals between January 2006 and December 2011 were invited to participate. Two age and sex matched controls per patient were recruited from individuals who were seen at the same department of neurology for non-neurodegenerative disorders. The participants were interviewed in a standardised telephone-interview. The questionnaire contained a complete occupational and residential history, and specific questions about potential risk and protective factors for Parkinson Disease. Among those were detailed questions about use of pesticide at work.

Results In total 444 Parkinson Disease patients and 876 controls were included in the analyses. We applied the ALOHA JobExposure-Matrix to assess exposure to pesticides in general and specifically to insecticides, to herbicides and to fungicides. Selfreported information allowed for a more detailed categorization of application and re-entry exposure. Preliminary analyses of the data show a slightly increased risk for Parkinson Disease for active application of herbicides and insecticides.

Conclusions Our results confirm earlier studies showing an elevated risk for Parkinson Disease after exposure to herbicides and insecticides. The final analyses will show the results of different levels of cumulative exposure resulting from application and reentry exposure.

\section{OCCUPATIONAL EXPOSURE TO MAGNETIC FIELDS AND ELECTRIC SHOCKS AND RISK OF ALS - ANALYSIS OF THE SWISS NATIONAL COHORT}

A Huss, Spoerri, Egger, Kromhout, Vermeulen. IRAS, Utrecht, The Netherlands

\subsection{6/oemed-2013-101717.283}

Background Previous studies have identified increased risks of amyotrophic lateral sclerosis (ALS) in electrical workers, but studies assessing risk in workers exposed to magnetic fields were ambiguous. Electric shocks have been hypothesised to be the relevant exposure, but potential risks from electric shocks have not been previously disentangled from those of magnetic fields. We aimed at analysing the association of ALS with both magnetic field exposure as well as risk of electric shocks at work.

Methods We used the Swiss National Cohort study, which is based on census information from 1990 and 2000 linked with mortality records from 2000-2008. High or medium versus low exposure to magnetic fields and risk of electric shocks was assigned to occupations using two job exposure matrices. Data of 4.7 million persons were analysed using Cox proportional hazard models and adjusted for age, sex, educational level, civil status, nationality, language region, degree of urbanity of the municipality and an area-based proxy for socioeconomic position.

Results During 2000-2008, 1313 persons with information on occupation died from ALS. Hazard ratios were around unity for persons exposed to magnetic fields or risk of electric shocks in either 1990 or 2000. In persons classified as medium or high exposed in both 1990 and 2000, a slight increase in mortality from ALS was observed for magnetic field exposures (HR 1.39, 95\% CI 1.03-1.87), but less so for electric shocks (HR 1.20, 95\% CI 0.88-1.65). When accounting for both exposures in the same model, magnetic fields had a similar HR $(1.36,95 \% \mathrm{CI}$ 0.99-1.89) as before, but the HR for electric shocks was attenuated to 1.07 (95\% CI $0.76-1.5$ ).

Conclusions We observed an association of long-term exposure to extremely low frequency magnetic fields and mortality from ALS, but not of risk of electric shocks and ALS.

\section{Session: 12. Late breaking abstracts}

\section{CANCER RISK AMONG TETRAFLUOROETHYLENE (TFE) SYNTHESIS AND POLYMERISATION WORKERS}

${ }^{1} \mathrm{D}$ C Consonni, ${ }^{2}$ Straif, ${ }^{3}$ Symons, ${ }^{4}$ Tomenson, ${ }^{5}$ Van Amelsvoort, ${ }^{6}$ Sleeuwenhoek, ${ }^{6}$ Cherrie, ${ }^{7}$ Bonetti, ${ }^{7}$ Colombo, ${ }^{8}$ Farrar, ${ }^{9}$ Carugno, ${ }^{9}$ Bertazzi. ${ }^{7}$ Fondazione IRCCS Ca' Granda Ospedale Maggiore Policlinico, Milan, Italy; ${ }^{2}$ International Agency for Research on Cancer, Lyon, France; ${ }^{3}$ DuPont Epidemiology Program, E. I. du Pont de Nemours and Company, Newark, DE, United States of America; ${ }^{4}$ Causation Ltd, Macclesfield, United Kingdom; ${ }^{5}$ University of Maastricht, CAPHRI, Maastricht, The Netherlands; ${ }^{6}$ Institute of Occupational Medicine, Edinburgh, United Kingdom; ${ }^{7}$ Solvay Specialty Polymers Italy SPA, Bollate, Italy; ${ }^{8}$ CCERT Ltd for Asahi Glass Company Chemicals Europe (AGCCE), Congleton, United Kingdom; ${ }^{9}$ ISCCO, Università degli Studi di Milano, Milano, Italy

\subsection{6/oemed-2013-101717.284}

Objectives Tetrafluoroethylene (TFE), a compound used for the production of fluorinated polymers including polytetrafluoroethylene (PTFE), increases liver and kidney cancer and leukaemia incidence in rats and mice. This is the first time the cancer risk in humans is comprehensively explored in a cohort mortality study.

Methods The study included all current PTFE production sites in Europe (Germany, Italy, The Netherlands and UK) and USA (New Jersey and West Virginia). The study cohort included workers ever employed in the period 1950-2002. A job-exposure matrix (1950-2002) was developed for TFE and ammonium perfluoro-octanoate (APFO), a chemical used in the polymerisation process. For each worker we calculated cumulative exposure to TFE and APFO. The mortality ascertainment covered the period 1950-2008. Standardised mortality ratios (SMR) and 95\% confidence intervals (CI) were calculated using national mortality rates as reference. 
Results Among 4,773 workers ever exposed to TFE, we found lower SMRs from most causes of death and increased SMRs for cancer of the liver (SMR 1.27; 95\% CI: 0.55, 2.51; 8 deaths) and kidney (SMR 1.44; 95\% CI: 0.69, 2.65; 10 deaths), and for leukaemia (SMR 1.48; 95\% CI: 0.77, 2.59; 12 deaths). A nonsignificant upward trend $(\mathrm{P}=0.24)$ by cumulative exposure to TFE was observed for liver cancer. TFE and APFO exposures were highly correlated, therefore their separate effects could not be disentangled.

Conclusions The pattern of findings in this large study substantially narrows the range of uncertainty on the possible cancer risk entailed by working in TFE synthesis and polymerisation, and justifies continuing efforts to minimise exposure, which has already dropped considerably over the years. However, the findings could neither conclusively confirm nor refute the hypothesis that TFE poses a carcinogenic risk to human beings. If a cancer hazard exists, then the risk is small, even in workers with relatively high exposure.

\section{NASAL MRSA CARRIAGE IN FARMERS STRONGLY ASSOCIATED WITH MRSA AIR LEVELS}

${ }^{1} \mathrm{M}$ E H Bos, ${ }^{2}$ van Cleef, ${ }^{3}$ Graveland, ${ }^{1}$ Dohmen, ${ }^{1}$ Dorado-Garcia, ${ }^{4}$ Duim, ${ }^{4}$ Verstappen, ${ }^{5}$ Kluytmans, ${ }^{4}$ Wagenaar, ${ }^{1}$ Heederik. ${ }^{1} / R A S$, Utrecht, Nederland; ${ }^{2}$ Laboratory for Medical Microbiology and Immunology, St. Elisabeth Hospital, Tilburg, Nederland; ${ }^{3}$ Centre for Infectious Disease Control, National Institute for Public Health, Bilthoven, Nederland; ${ }^{4}$ Department of Infectious Diseases and Immunology, Faculty of Veterinary Medicine, Utrecht, Nederland; ${ }^{5}$ Department of Medical Microbiology and Infection prevention, VU University, Amsterdam, Nederland

\subsection{6/oemed-2013-101717.285}

Objectives MRSA carriage prevalence is as high as 60\% in livestock farmers in the Netherlands. Human carriage was associated with carriage in animals. However, animal-to-human transmission and especially airborne exposure are poorly studied. We determined MRSA air levels and a possible dose-response relationship with nasal MRSA carriage in farmers.

Methods Human MRSA carriage and stable MRSA air levels were assessed in three independent populations of 38 assumed frontrunner pig farms (A), 50 random pig farms (B), and 49 veal calf farms (C). Farmers were identified as human participants spending on average $\geq 20$ hours/week working on the farm. Per farm 1-6 electrostatic dust collectors (EDCs) were placed. Nasal swabs were taken from farmers and analysed for presence of MRSA by culturing and PCR. EDCs were analysed by qPCR, which resulted in an equivalent of the number of colony forming units (CFUeq) per EDC.

Results Mean MRSA air levels were lowest on veal farms and highest on pig farms. Generally, the average MRSA air levels corroborated with the MRSA nasal carriage prevalence in the three populations. Mean MRSA/EDC/farm for population A was 225 CFUeq (95\%CI: 170-281); for population B it was 385 CFUeq (95\%CI: 239-530), and for population $\mathrm{C}$ it was 59 CFUeq (95\%CI:26-93). Nasal MRSA carriage prevalences were 53\% for population A, $72 \%$ for population $\mathrm{B}$, and $31 \%$ for population C. Working hours ranged from 20-80 hours/week, with means of 48, 46, and 39 hours for population A, B, and C, respectively.

In a multivariate pooled analysis, a strong association between MRSA carriage and the mean MRSA concentration (expressed as $\log (\mathrm{MRSA}) / \mathrm{EDCs} /$ farm $)$ was shown $(\mathrm{RR}=1.37 ; \mathrm{p}=0.0008)$, independent of the association with working hours $(\mathrm{RR}=1.01$; $\mathrm{p}=0.03$ ) and adjusted for age, sex and smoking.
Conclusions Results suggest an important role of MRSA transmission through air, which has not been established earlier.

\section{REDUCED HEALTHCARE-ASSOCIATED INFECTIONS FOLLOWING A UK WIDE CAMPAIGN PROMOTING HAND WASHING COINCIDED WITH INCREASED IN CONTACT DERMATITIS IN HEALTHCARE WORKERS}

S J Stocks, Turner, McNamee, Carder, Agius. University of Manchester, Manchester, United Kingdom

\subsection{6/oemed-2013-101717.286}

Objectives Over the past decade there has been increasing concern among the public and government about high rates of healthcare associated infections and low levels of hand hygiene compliance. In response the "Cleanyourhands" campaign was rolled out from 2004 in all acute NHS hospital trusts. A national level evaluation of this intervention found a substantial increase in the use of hand cleaning products in acute trusts between 2004 and 2008, which was associated with a reduction in meticillin resistant $S$ aureus and $C$ difficile infections ${ }^{1}$. This study aims to compare the increased usage of hand hygiene products ${ }^{1}$ in acute NHS trusts with changes in the incidence of CD attributed to hand washing in healthcare workers.

Methods Reports of occupational CD to a surveillance scheme by dermatologists and occupational physicians (OPs) were analysed, using a prospective interrupted time series design with time periods matching those used in the evaluation of the "Cleanyourhands" intervention ${ }^{1}$. Comparisons were made between reports attributed to frequent hand washing and other causal agents, to mitigate bias arising from the voluntary nature of the reporting scheme.

Results The incidence of CD attributed to hand washing was significantly increased relative to all other causes in healthcare workers following the "Cleanyourhands" campaign (statistical interaction;95\% CIs: dermatologists 2.19; 1.62 - 2.96, OPs $2.44 ; 1.15-5.18)$. The increase reported by dermatologists was predominantly irritant $\mathrm{CD}(2.58 ; 1.74-3.81)$ rather than allergic CD (1.04; $0.38-2.84)$.

Conclusion The increase in irritant CD reported by dermatologists, and all CD by OPs, is consistent with the increase in use of hand cleaning agents following the "Cleanyourhands" campaign. Attention should be paid to the adverse effects of frequent hand washing as well as prevention of infections.

${ }^{1}$ Stone et al BMJ 2012;344:e3005 doi: 10.1136/oemed-2013101717.287

\section{OCCUPATIONAL EXPOSURES AND AMYTROPHIC LATERAL SCLEROSIS MORTALITY IN A LARGE PROSPECTIVE COHORT}

${ }^{1} \mathrm{~T}$ Koeman, ${ }^{1}$ Slottje, ${ }^{2}$ Leo, ${ }^{1}$ Huss, ${ }^{3}$ Peters, ${ }^{4}$ Bausch-Goldbohm, ${ }^{2}$ van der Brandt, ${ }^{1}$ Kromhout, ${ }^{1}$ Vermeulen. 'IRAS, Utrecht, Nederland; ${ }^{2}$ Maastricht University, Maastricht, Nederland; ${ }^{3}$ Western Australian Institute for Medical Research, Nedlands, Australia; ${ }^{4}$ TNO, Leiden, Nederland

\subsection{6/oemed-2013-101717.288}

Objectives This study aims to study multiple occupational exposures and their possible associations with Amyotrophic Lateral Sclerosis (ALS) mortality within the Netherlands Cohort Study (NLCS).

Methods For this case-cohort analysis, 120,852 persons aged 55 to 69 years at time of enrollment in 1986 were followed up 